\title{
NMR Studies of the Electronic Structure of Benzocycloheptatriene Ions. Absence of Ion Pair Effects in Carbenium Salts
}

\author{
Ingmar Sethson, Dan Johnels and Ulf Edlund* \\ NMR Research Group, Department of Organic Chemistry, University of Umeå, S-901 87 Umeå, Sweden
}

\begin{abstract}
Sethson, I., Johnels, D. and Edlund, U., 1990. NMR Studies of the Electronic Structure of Benzocycloheptatriene Ions. Absence of Ion Pair Effects in Carbenium Salts. - Acta Chem. Scand. 44: 1029-1031.

An NMR chemical shift study of benzocycloheptatrienyl ions has shown that ion pair effects are insignificant for the estimated charge distribution. The carbon shifts induced by varying the solvent or by the presence of various counterions are small compared with the shifts induced for the corresponding paratropic anion and the isoelectronic indenide system.
\end{abstract}

It is well established that ion pair changes of delocalized alkali metal carbanion salts significantly perturb the charge distribution of the carbanion system. Such field and polarization effects are reflected in induced NMR shifts, especially in induced ${ }^{13} \mathrm{C}$ NMR shifts. ${ }^{1}$ In the case of delocalized carbocations one would expect such changes to be suppressed, when the counterions are bulky and therefore have a limited tendency to form specific solvates. However, the possibility of ion pairing effects for carbenium salts can not safely be excluded. It has been suggested that such effects can rationalize the variation observed in hydride transfer reactions of alkyl carbocations in superacidic solutions. ${ }^{2}$ So far, no spectroscopic evidence for the presence or absence of such ion pairing effects has been presented. In a ${ }^{1} \mathrm{H}$ NMR study of the tropylium ion a negligible ion pairing effect was suggested. ${ }^{3}$ This delocalized cation, however, is not suitable for such studies, since $\pi$-polarization changes are not expected due to molecular symmetry.

In this study the benzocycloheptatriene $(\mathrm{BCH})$ cation was used as a model system. This cation can be compared to the isoelectronic indenide compound. ${ }^{4} \mathrm{NMR}$ and molecular orbital data (HMO) are also presented for a reference molecule, the $\mathrm{BCH}$ anion, in order to build a complete electronic picture of the $\mathrm{BCH}$ system.

The anion and cation of BCH (Fig. 1) have $4 n \pi$ and $(4 n+2) \pi$ electron systems, respectively. The expected paratropicity and diatropicity of the two systems have earlier been confirmed. ${ }^{5}$ MNDO calculations of the $\mathrm{BCH}^{-}$ system resulted in two structures of $C_{\mathrm{s}}$ and $C_{2 \mathrm{v}}$ symmetry, both of approximately the same heat of formation. ${ }^{6}$ The $\mathrm{C} 9-\mathrm{C} 9 \mathrm{a}$ and $\mathrm{C} 5-\mathrm{C} 4 \mathrm{a}$ bonds are essentially single bonds, especially so in the $C_{2 \mathrm{v}}$ structure. The two $6 \pi$-electron systems are therefore, to a large extent, repulsive. ${ }^{6}$

In this report, ${ }^{13} \mathrm{C}$ NMR chemical shifts have been used to estimate ion pair effects on charge distribution. Carbon shifts are less sensitive than proton shifts, with regard to

${ }^{*}$ To whom correspondence should be addressed.

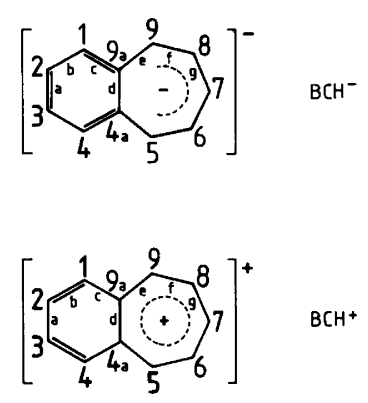

changes in ring susceptibilities. The ${ }^{13} \mathrm{C}$ shift data reported in Table 1 were determined in various solvents, and by varying temperature and counterions.

The data for $\mathrm{BCH}^{+}$indicate that the variation in the ${ }^{13} \mathrm{C}$ chemical shifts is only to within $1 \mathrm{ppm}$. This is a considerably smaller range than for the observed shift variance for the isoelectronic indenide system. Significantly, ${ }^{13} \mathrm{C}$ chemical shift changes, $\Delta \delta_{\mathrm{C}}$ ca. $6 \mathrm{ppm}$, have been observed for the delocalized indenide system by varying the solvent. ${ }^{4}$ In the case of $\mathrm{BCH}^{+}$, no systematic pattern in the data is observed that may indicate any changes in the degree of polarization of the benzocycloheptatrienium ion. The change from an acidic to an organic medium, dichloromethane, did not induce significant shift changes. Additional support for the absence of ion pairing effects comes from a comparison with the solid-state NMR data of $\mathrm{BCH}^{+} \mathrm{ClO}_{4}{ }^{-}$. The shift values are almost identical with the solution values (Table 1).

The situation is quite different for $\mathrm{BCH}^{-}$. The shielding of the carbon atom of highest charge in the seven-membered ring is increased, when solvent effects favour intimate ion pairs. Significant counterion-induced charge polarization is thus clearly manifested.

In order to estimate the charge distribution of the $\mathrm{BCH}$ ions, one has to rely on either MO calculations or experimental estimates, in our case charge values based on the induced carbon shifts. Provided that one can account for 
Tab/s 1. NMR chemical shifts of benzocycloheptatrienium and benzocycloheptatrienide ions on variation solvent, temperature and counterion. $^{a}$

\begin{tabular}{llllllll}
\hline Temp. ${ }^{\circ} \mathrm{C}$ & Solvent & Counterion & \multicolumn{3}{l}{ Position } & & \\
\cline { 3 - 6 } & & 5,9 & 7 & $4 a, 9 a$ & 6,8 & 2,3 & 1,4 \\
\hline
\end{tabular}

\section{Carbocation}

$+20 \quad \mathrm{HF}$

$+20$

HF

$+20$

$+20$

$-30$

$\mathrm{HBr}$

$\mathrm{HSO}_{3} \mathrm{Cl}$

$\mathrm{CH}_{2} \mathrm{Cl}_{2}$

$F^{-b}$

$\mathrm{Cl}^{-b}$

$\mathrm{Br}^{-}$

$\mathrm{SO}_{3} \mathrm{Cl}^{-}$

$\mathrm{CH}_{2} \mathrm{Cl}_{2}$

$\mathrm{ClO}_{4}^{-}$

$-30$

$-30$

$\mathrm{FClSO}_{2}$

$\mathrm{BF}_{4}$

$-30$

$\mathrm{HSO}_{3} \mathrm{Cl}$

$\mathrm{SO}_{3} \mathrm{Cl}^{-}$

$-60$

$\mathrm{FCISO}_{2}$

$\mathrm{SO}_{3} \mathrm{Cl}^{-}$

$\mathrm{SO}_{3} \mathrm{Cl}^{-}$

Solid

$\mathrm{ClO}_{4}^{-c}$

$\begin{array}{cc}160.9 & 158.5 \\ 160.9 & 158.3 \\ 160.7 & 158.5 \\ 161.1 & 158.3 \\ 161.5 & 158.8 \\ (9.45) & (8.95) \\ 161.3 & 158.7 \\ (9.50) & (9.05) \\ 161.3 & 158.7 \\ (9.45) & (9.00) \\ 161.2 & 158.4 \\ 161.5 & 158.8 \\ 161 & 158\end{array}$

144.9
144.7
144.6
145.2
145.0
$(8.65)$
145.0
$(8.70)$
145.4
$(8.65)$
145.1
145.4
145

141.2
141.1
141.3
141.2
141.7
$(8.30)$
141.6
$(8.40)$
141.4
$(8.45)$
141.3
141.6
140

139.1

139.1

137.8

$139.1 \quad 137.9$

$139.6 \quad 138.2$

139.6

$(-)$

139.5

$(-)$

139.8

(-)

139.6

139.9

140

138.4

(8.55)

138.3

(8.60)

138.4

(8.65)

138.3

138.5

140

\section{Carbanion}

$-25$

THF- $d_{8}$

$\mathrm{Li}^{+}$

$\begin{array}{cc}109.5 & 86.2 \\ (0.1) & (-0.5) \\ 108.8 & 84.2 \\ (0.7) & (0.0) \\ 108.9 & 90.3\end{array}$

$\begin{array}{cc}161.7 & 151.0 \\ (-) & (1.9) \\ 159.3 & 150.2 \\ (-) & (2.5) \\ 160.7 & 151.2\end{array}$

127.2
$(3.9)$
127.0
$(4.3)$
128.3

123.1

(2.9)

123.9

aThe NMR spectra of $\mathrm{BCH}^{+}$were obtained on a Bruker AC-P 250 spectrometer using an external lock as the reference. The NMR spectra of the anions were obtained on an AM 500 spectrometer using cyclohexane as an internal reference (27.7 ppm). The values in parentheses are the proton chemical shifts when recorded. ${ }^{b}$ These salts were not stable in acidic solution and decomposed within a few hours to the covalently halo-substituted benzocycloheptenes. 'The resonances of the CP/MAS spectrum were broadened, probably due to dipolar coupling to the oxygens of the perchlorate anion. The three high-field resonances are merged into one broad peak around $140 \mathrm{ppm}$.

paramagnetic contributions arising from differences in HOMO-LUMO gaps in the ion and its reference molecule, 'experimentally' derived charges are often prefered to MO-calculated charges. ${ }^{7}$ The MO-calculated values either neglect counterion or solvent effects or describe them only poorly.

The sum of the induced carbon shifts on going from the cation to the anion of $\mathrm{BCH}$, leads to a sensitivity-to-charge ratio $\left(K_{\mathrm{C}}\right)$ of $89 \mathrm{ppm}$ per electron. This value is much smaller than the commonly quoted value of $160 \mathrm{ppm}$ per electron for cyclic aromatic ions. We have previously demonstrated that low $K_{\mathrm{C}}$ values are observed when the reduction affords a paratropic carbanion. ${ }^{7 a}$ The low shift/charge factor is thus a confirmation of the paratropicity of the $\mathrm{BCH}$ anion, i.e. a decreased HOMO-LUMO gap relative to the gap of $\mathrm{BCH}^{+}$. The paramagnetic contributions related to the differences in tropicity can be accounted for as previously described. ${ }^{7}$ The carbon shifts, corrected for such anisotropy changes, are then used to calculate the experimental charges (Table 2). The overall agreement between calculated and experimental charges is particularly satisfying for the diatropic $\mathrm{BCH}^{+}$, where ion pairing is suggested to be insignificant. For $\mathrm{BCH}^{-}$, however, the estimated 'experimental' charge is higher than the calculated one at the carbon atom of highest charge in the seven-
Table 2. Experimental $\pi$-charges, derived from ${ }^{13} \mathrm{C}$ chemical shifts together with $\mathrm{HMO} \pi$-charges.

\begin{tabular}{|c|c|c|c|c|c|c|}
\hline & \multirow[t]{2}{*}{$\mathrm{BCH}$} & \multicolumn{5}{|c|}{ Position } \\
\hline & & 5,9 & 7 & 6,8 & 2,3 & 1,4 \\
\hline$\delta^{13} C^{a}$ & + & 161.1 & 158.3 & 141.2 & 139.6 & 138.2 \\
\hline $\begin{array}{l}\delta^{13} \mathrm{C} \\
\text { (corr.) }\end{array}$ & + & 161.7 & 159.0 & 141.9 & 140.3 & 138.9 \\
\hline$\delta^{13} C^{c}$ & - & 109.5 & 86.2 & 151.0 & 127.2 & 123.1 \\
\hline $\begin{array}{l}\delta^{13} \mathrm{C} \\
{\text { (corr. })^{b}}^{b}\end{array}$ & - & 99.0 & 75.5 & 140.5 & 116.7 & 112.6 \\
\hline $\begin{array}{l}\exp . \varrho_{\pi}{ }^{d} \\
\text { (corr.) }\end{array}$ & + & +0.19 & +0.17 & +0.08 & +0.07 & +0.06 \\
\hline $\mathrm{HMO} \varrho_{\pi}$ & + & +0.19 & +0.18 & +0.08 & +0.05 & +0.03 \\
\hline $\begin{array}{l}\exp . \varrho_{\pi}^{d} \\
\text { (corr.) }\end{array}$ & - & -0.21 & -0.39 & +0.09 & -0.08 & -0.11 \\
\hline $\mathrm{HMO} \varrho_{\pi}$ & - & -0.26 & -0.29 & +0.07 & -0.05 & -0.12 \\
\hline
\end{tabular}

${ }^{a}{ }^{13} \mathrm{C}$ chemical shifts of $\mathrm{BCH}^{+}$in $\mathrm{HSO}_{3} \mathrm{Cl}$ at $+20^{\circ} \mathrm{C}$. ${ }^{b}$ When using benzene as a shift reference, the calculated ring current anisotropy $X_{H}$ is -4.4 and $0.4 \mathrm{ppm}$ for the anion and cation, respectively. The corresponding $X_{C}$ values, 10.5 and $-0.7 \mathrm{ppm}$, are subtracted from the experimental shift values. See Ref. 7 for details. ${ }^{13} \mathrm{C}$ chemical shiffts of lithium $\mathrm{BCH}^{-}$in THF- $d_{8}$ at $-25^{\circ} \mathrm{C}$. ${ }^{d} \mathrm{Charges}$ calculated using corrected shift values and 180 and $134 \mathrm{ppm}$ per electron for the cation and anion, respectively. ${ }^{7}$ Benzene is used as a reference for zero charge. 
membered ring. This is as expected, if charge polarization to the seven-membered ring is significant, i.e. in a contact ion pair. One may also note that charge alternation is more pronounced in the paratropic $\mathrm{BCH}^{-}$system.

The present results lead to the conclusion that ion pair effects are insignificant for a delocalized carbenium ion such as the benzocycloheptatrienium ion. The carbon shifts induced by the presence of various counterions or by varying the solvent are very small compared with the shifts induced for the corresponding anion and the isoelectronic indenide system.

\section{Experimental}

The ${ }^{13} \mathrm{C}$ NMR spectra were assigned by using $\mathrm{C}, \mathrm{H}$-correlated 2D NMR spectroscopy. Identification of the proton resonances of the carbanion was performed by $\mathrm{DQF}-$ COSY 2D due to the low concentration and interference from larger signals. Benzocycloheptatrienium ions were prepared by treatment of benzocycloheptatrien-5-ol with acid. $^{8}$

Benzocycloheptatrienyl anions were prepared by treatment of a mixture of $5 \mathrm{H}$ - and $7 \mathrm{H}$-benzocycloheptatriene with trimethylsilylmethyl-lithium (or -potassium) at $-78^{\circ} \mathrm{C}$ in THF- $d_{8}$ following the procedure for the preparation of benzyl anions. ${ }^{9}$

Benzocycloheptatrien-5-ol was prepared by treating benzocycloheptatrien-5-one with $\mathrm{LiAlH}_{4}$ in diethyl ether $\left(-17^{\circ} \mathrm{C}\right) .^{10}$ The benzocycloheptatriene mixture was prepared by treatment of benzocycloheptatrien-5-one with diisobutylaluminium hydride in hexane and refluxing overnight, following the procedure for the preparation of phe- nalene from phenalenone. ${ }^{11}$ The product was purified by column chromatography using Florisil (mesh 200-300) with hexane as the eluent. Benzocycloheptatrien-5-one was prepared from the commercially available 6,7,8,9-tetrahydrobenzocycloheptatrien-5-one (benzosuberone). ${ }^{12}$

Acknowledgements. Grants from the Swedish Natural Science Research Council to U.E. are gratefully acknowledged.

\section{References}

1. Young, R. N. Prog. NMR Spectrosc. 12 (1979) 261.

2. Arnett, E. M. and Hofelich, T.C. J. Am. Chem. Soc. 104 (1982) 3522.

3. Cox, R. H., Harrison, L.W. and Austin, W. K. J. Phys. Chem. 77 (1973) 200.

4. Eliasson, B., Johnels, D., Wold, S. and Edlund, U. Acta Chem. Scand., Ser. B 36 (1982) 155.

5. Staley, S. W. and Orvedal, A. W. J. Am. Chem. Soc. 95 (1973) 3382.

6. Glidewell, C. and Lloyd, D. Tetrahedron 40 (1984) 4455.

7. (a) Eliasson, B., Edlund, U. and Müllen, K. J. Chem. Soc., Perkin Trans. 2 (1986) 937; (b) Eliasson, B., Johnels, D., Sethson, I., Edlund, U. and Müllen, K. J. Chem. Soc., Perkin Trans. 2. In press.

8. Rennhard, H. H., Heilbronner, E. and Eschenmoser, A. Chem. Ind. (London) (1955) 415.

9. Hart, A. J., O'Brien, D. H. and Russell, C. R. J. Organomet. Chem. 72 (1974) C 19.

10. Rennard, H. H., Di Modica, G., Simon, W., Heilbronner, E. and Eschenmoser, A. Helv. Chem. Acta 40 (1957) 957.

11. Boudjok, P. and Johnson, P. D. J. Org. Chem. 43 (1978) 3979.

12. Collington, E. W. and Jones, G. J. Chem. Soc. C (1969) 2656.

Received March 7, 1990.

\footnotetext{
KEM15: e: ?
} 